Para enlazar con este artículo / To link to this article:

http://dx.doi.org/10.14198/fem.2019.33.07

Para citar este artículo / To cite this article:

Martínez-García, Patricia. "Cuando las mujeres acceden al poder. discriminaciones y resistencias en las comunidades marineras». En Feminismo/s, 33 (junio 2019): 171-196. Dosier monográfico: Diálogos entre la democracia participativa y la interseccionalidad. Construyendo marcos para la justicia social, coords. Patricia Martínez-García y Jone Martínez-Palacios, DOI: 10.14198/fem.2019.33.07

\title{
CUANDO LAS MUJERES ACCEDEN AL PODER. DISCRIMINACIONES Y RESISTENCIAS EN LAS COMUNIDADES MARINERAS
}

\author{
WHEN WOMEN REACH POSITIONS OF POWER. \\ DISCRIMINATIONS AND RESISTANCES IN FISHERIES \\ COMMUNITIES
}

\author{
Patricia MARTÍNEZ-GARCÍA \\ Aradia Cooperativa y Universidad del País Vasco \\ patmartinez.garcia@gmail.com \\ https://orcid.org/0000-0003-3894-6881
}

\section{Resumen}

Las trabajadoras del mar y, en concreto, las mariscadoras a pie, se han convertido en un agente fundamental en el impulso de cambios en las comunidades pesqueras. Desde su profesionalización en Galicia en los años 90, se han sucedido transformaciones en su posición económica, política y social. Desde la marginación y la falta de reconocimiento se han consolidado como referentes de nuevas formas de organización y dinamización de sus comunidades. De manera que el objetivo de este trabajo es analizar las experiencias de participación de las mariscadoras a pie en las organizaciones pesqueras. A través de una metodología cualitativa, sustentada en el análisis documental y en la realización de entrevistas en profundidad, se atiende a las discriminaciones que han vivido y a las estrategias que han desarrollado para sobrevivir, muchas veces, en un entorno hostil.

Palabras clave: discriminación de género, mariscadoras a pie, organizaciones pesqueras, participación política, resistencias.

Los contenidos de la revista se publican bajo una licencia de Creative Commons Reconocimiento 4.0 Internacional (CC BY 4.0)

Feminismo/s 33, junio 2019, pp. 171-196 


\begin{abstract}
Women seafarers, in particular, shellfish gatherers, have become key players to promote changes in fisheries communities. From their professionalization in Galicia in the '90, have experienced economical, political and social changes: From marginalization and lack of recognition have become references of organization and revitalization of their communities. Thus, this article will analyze the shellfish gatherers experiences of participation en fishing organizations. Through an qualitative research methodology, using documental analysis and in-depth interviews, it examines how these women are discriminated and how they face them to survive in an hostile environment.
\end{abstract}

Keywords; Gender discrimination, shellfish gatherers, fisheries organizations, political participation, resistances.

\title{
1. INTRODUCCIÓN
}

Las comunidades pesqueras han sido testigos de las transformaciones en las posiciones de poder de las trabajadoras del mar. Los cambios en sus condiciones laborales han venido acompañados de un mayor reconocimiento y visibilidad social, de una mayor autonomía económica y de una mayor influencia política (Martínez-García, Empoderamiento femenino). A este respecto, Galicia se constituye como epicentro de estas alteraciones, debido a la importancia que la pesca juega en este territorio. Con sus aproximadamente 1500 kilómetros de costa, este sector no solo juega un papel relevante en lo económico, sino también como configurador de su idiosincrasia (Marugán, La organización) y de las relaciones de poder en su interior, también de género, como iremos viendo a lo largo de este artículo.

Esta magnitud se refleja en la proporción de empleos creados en torno al ámbito pesquero. Según datos del Instituto Social de la Marina, Galicia es la comunidad con más personas afiliadas al Régimen Especial del Mar (REM) con más de 20.500 -muy por encima de la segunda, Andalucía, que cuenta con unas 7.500 filiaciones-. De ellas, las mujeres ocupan el 24\%-unas 5.200lo que las convierte en el $55 \%$ de las trabajadoras del mar en toda España. No obstante, hay que tener en cuenta que las estadísticas invisibilizan la presencia femenina y su papel histórico en las comunidades (García Negro y Zotes 4): 
En el caso de Galicia, existe una relación histórica con el mar; las mujeres de la costa son protagonistas de la actividad pesquera, adquiriendo una importancia socioeconómica decisiva en el sector pesquero ya que participan en toda la cadena productiva, desde el subsector extractivo hasta la comercialización final del producto [...] Todas las actividades pesqueras dependen, en mayor o menor medida, de esta relación histórica creadora de todo tipo de enlaces de naturaleza económica, donde las mujeres fueron protagonistas: pescando, cultivando, transformando, vendiendo y transmitiendo saberes (conocimiento) para repetir generación tras generación esa relación íntima entre habitantes y mar.

Aunque, como se recoge en la cita, las mujeres han sido protagonistas de toda la actividad pesquera, su distribución en las diferentes tareas está condicionada por el género. A este respecto, la división sexual del trabajo en estos entornos está influida por la frontera física entre la tierra y el mar (Broullón, La politica sexual; Marugán, La organización). El Diagnóstico sobre la situación de la mujer en el Sector Pesquero y Acuícola, elaborado por la Red Española de Mujeres del Sector Pesquero (REMSP), pone de evidencia esta diferencia de representatividad por ramas. Por poner algunos ejemplos, en la extracción a bordo de las embarcaciones la presencia femenina es ínfima, mientras que son mayoría en todas aquellas actividades de apoyo (rederas, empacadoras o neskatillas ${ }^{1}$, de transformación y comercialización ${ }^{2}$.

1. Las neskatillas son las mujeres encargadas de la descarga del pescado del barco y de su venta. Por su parte, las empacadoras pesan, clasifican y ordenan las cajas descargadas. La actividad de ambos colectivos se concentra en los puertos vizcaínos de Bermeo y Ondarroa.

2. En los últimos años, la REMSP ha hecho un esfuerzo por diagnosticar la situación de las mujeres en los distintos sectores: Rederas, un oficios desconocido (2012); Diagnóstico sobre la situación profesional de las mujeres en el oficio del marisqueo en España (2016); Diagnóstico sobre la situación profesional del colectivo de neskatillas y empacadoras (2016); Diagnóstico sobre la situación de la mujer en la acuicultura (2016); Diagnóstico sobre la situación de la mujer en la pesca extractiva (2017); Diagnóstico sobre la situación de la mujer en la industria de transformación de productos pesqueros y acuícolas (2018), y el Diagnóstico sobre la situación de la mujer en la comercialización de productos pesqueros y acuícolas (2018). Profundizar en cada uno de estos colectivos sobrepasa los objetivos de este artículo, por lo que, para ampliar esta información, se recomienda consultar la página: <https://www.mapa.gob.es/es/pesca/temas/red-mujeres/analisis_y_diagnosticos. aspx $>$

Feminismo/s 33, junio 2019, pp. 171-196 
Este reparto de tareas tiene consecuencias en la distribución de espacios y, con ello, en las condiciones laborales, en la visibilidad de los oficios y en el ejercicio del poder en estas comunidades. Los colectivos feminizados son aquellos que ocupan, sobre todo, los empleos ejercidos por cuenta propia y principalmente manuales, afectados por la alta temporalidad, con salarios bajos y jornadas indefinidas (Gago y Ardora; Federación Galega de Confrarías de Pescadores; REMSP, Diagnóstico sobre la situación de la mujer). Es decir, las comunidades marineras encarnan en el binomio mar-tierra el dualismo jerárquico en el que se sostienen las desigualdades de género (Broullón, Culturas marítimas; Fundación Mujeres; Martínez-García, Empoderamiento femenino).

\subsection{El mar y la tierra como representaciones de lo público y lo privado}

Esta división sexual del trabajo reproduce otras lógicas de dominación sustentadas en el género, pues nuestras sociedades se han moldeado en torno a una contraposición entre lo masculino y lo femenino que encarnan valores diferenciados: cultura-naturaleza, razón-emoción, público-privado, producción-reproducción, etc. (Amorós, Hacia una crítica; Marçal; Pateman). En palabras de Elena Salas y Nieves Salobral (126):

Mientras que Edipo es el varón de la familia y por tanto el logos, lo público, el jerarca, lo político; Electra es la hija y los valores que el Patriarcado impone como lo femenino: el ámbito de lo doméstico, los cuidados, los afectos, lo irracional y depositario del honor familiar [...] Mientras que Electra existe como sujeto en función de la necesidad familiar, Edipo es sujeto constituido en sí mismo [...].

Como se desprende de este fragmento, esta dualidad no es horizontal, sino jerárquica. El primer par (cultura, razón, público, producción) se convierte en el criterio de valor, mientras el segundo (naturaleza, emoción, privado, reproducción) es devaluado (Fraser, Dilemas). Lo masculino y lo femenino son así marcados con diferentes grados de reconocimiento. A este respecto, la figura del iceberg utilizada por la economía feminista es muy ilustrativa. Como explica Amaia Pérez Orozco (La economía 238), la zona visible del bloque de hielo se corresponde con lo público y lo monetizado, mientras que la invisible lo hace con lo privado, donde se localiza el trabajo doméstico y de cuidados, así como «el voluntario o de participación ciudadana, el trabajo 
en redes sociales, o aquellas otras actividades que se enmarcan en ocasiones bajo el nombre de trabajo de subsistencia».

En el caso de las comunidades marineras, esta figura del iceberg se bosquejaría en torno a una parte visible, que sería el mar, y una invisible, que sería la tierra. Como observa en su análisis Esmeralda Broullón (Culturas marítimas; La política sexual) el mar se ligaría al heroísmo y al riesgo y, con ello, a la masculinidad. Y todas aquellas aportaciones de las mujeres quedan deslucidas pese a ejercer tareas imprescindibles para la supervivencia familiar como es el atar, el avituallamiento de enseres, la carga y la descarga o la extracción de bivalvos. En este sentido, se olvida que «las mujeres marítimas han sido, son y seguirán siendo un elemento clave del mantenimiento y sostenimiento de la cultura pesquera» y también del medio (Marugán, E colleron 9).

En definitiva, en las sociedades marítimas también se reproduce ese mito de la autonomía y la independencia plena en una concepción androcéntrica del ideal ciudadano y trabajador (Amorós, Tiempo de; Fraser, Fortunas; Pérez Fuentes), mientras se invisibiliza todo aquello que sostiene la vida, que cuida los cuerpos y el entorno (Carrasco; Izquierdo; Herrero, Pascual y González Reyes; Pérez Orozco, Subversión feminista; Vega, Martínez Buján y Paredes Chauca).

Sin embargo, este par mar/tierra contiene ciertas peculiaridades que desgastan, en cierto sentido, la citada división de espacios tradicional. Por un lado, el hecho de que los hombres pasen (cortas o largas) temporadas en alta mar provoca que deleguen en las mujeres todo el trabajo de supervivencia diaria, comunitario, y por supuesto, de cuidados (Broullón, La política sexual; Del Valle; Fundación Mujeres). Algo que impacta de lleno en la vida de las mujeres. Pues, como tan acertadamente definió la poeta Rosalía de Castro al hablar de «viudas de vivos», estas ausencias posibilitaron la extensión de la idea de matriarcado en las costas gallegas: mujeres solas, de una extremada fortaleza y que sostenían la vida durante las ausencias de sus maridos (Broullón, La política sexual; Martínez-García, Empoderamiento femenino). Por otro lado, aunque, como se ha dicho, los trabajos costeros feminizados estén muy vinculados a la parte baja del iceberg, lo cierto es que se desempeñan en lo público (en puertos, playas, plazas...) y, con ello, estas mujeres tenían una mayor presencia física en espacios que, en otros contextos estaban reservados para los hombres. Una presencia que derivó en una construcción de una

Feminismo/s 33, junio 2019, pp. 171-196 
feminidad alejada de los patrones de debilidad y discreción más tradicionales (Del Valle).

Si bien las fronteras físicas entre lo público y lo privado se diluyen en las comunidades pesqueras (no sin sanciones simbólicas, como veremos más adelante), se mantienen sus consecuencias, que se pueden resumir en una explotación no cuantificada del trabajo de las mujeres. Como nos recuerdan Yayo Herrero, Marta Pascual y González Reyes (43): «No es una paradoja. Ni despiste ni ceguera involuntaria. Lo que nos sostiene se invisibiliza para que pueda ser fácilmente apropiable. Normalizar un reparto profundamente desigual permite perpetuarlo». De esta manera, las trabajadoras del mar experimentan las dinámicas en las que convergen las lógicas de dominación patriarcal y capitalista: (1) La naturalización en las mujeres de las tareas ligadas a lo reproductivo; (2) La perpetuación de un entramado cultural y social que desvaloriza y oculta todo aquello que tiene que ver con este ámbito, y (3) La interiorización de las propias mujeres de esta obligatoriedad. A continuación, concretaremos en el marisqueo todas estas cuestiones.

\subsection{De la marginalidad y precariedad a la dignificación y reconocimiento}

Según se recoge en el artículo 27 de la Ley de Pesca de Galicia de 2008, el marisqueo se define como:

El ejercicio de la actividad extractiva, desarrollada a pie o desde embarcación, en la zona marítima o marítimo-terrestre dirigida de modo exclusivo y con artes selectivas y específicas a la captura de una o varias especies de moluscos, crustáceos, tunicados, equinodermos y otros invertebrados marinos, con fines de comercialización.

En esta definición vemos cómo es una actividad que se puede realizar a pie o a flote. Como se desprende de lo mencionado anteriormente, el marisqueo desde embarcación lo ejercen casi en exclusiva los hombres, mientras el que se realiza en las playas lo desarrollan las mujeres. Aunque el porcentaje ha ido descendiendo progresivamente, según los datos del Instituto Galego de Estadística (IGE) de 2018, la Xunta de Galicia otorgó 3792 Permisos de 
Explotación Marisquera (Permex) ${ }^{3}$, de los cuales el 72\% corresponden a mujeres. Una cifra que ha disminuido sensiblemente -en los 90 el porcentaje era del 95\%-debido a la creciente incorporación masculina a partir de la crisis económica de 2010 y de la mejora de las condiciones laborales y económicas conseguidas (REMSP, Diagnóstico de la situación...en el marisqueo).

En otras palabras, las condiciones en las que se ejercía el marisqueo a pie no siempre fueron dignas. Antes de la profesionalización del oficio, prevalecía una idea de que los recursos marisqueros eran de todo el mundo. Y, con ello, cualquiera disponía de los mismos (Mahou, Santasmarinas), sin normas de actuación que regularan la extracción e independientemente de los perjuicios económicos y ecológicos que se sucedían de la sobreexplotación de los arenales (Marugán, Estrategias Laborales). Esta forma de ejercer la actividad estaba también marcada por la marginalidad (Mahou; Marugán, E colleron), como queda reflejado en la propia definición recogida en el Plan de Ordenación de los Recursos Pesqueros y Marisqueos de 1993 (331). Según esta normativa el marisqueo se caracteriza por:

una mano de obra excesiva, una ausencia generalizada de formación profesional y cultura agravada por tratarse de un colectivo de edad media avanzada y a la que no es ajena la tradicional dificultad de las mujeres, componente mayoritario del subsector, para acceder a la formación, unos métodos de extracción manuales poco eficaces, un escaso interés del colectivo por mejorar se rendimiento[...].

Siguiendo con la descripción del marisqueo en los años previos a su regularización, se puede completar la fotografía hablando de: a) salarios bajos; b) alta temporalidad e irregularidad en el ejercicio de la actividad; c) dependencia de intermediarios (armadores o comerciantes); d) inexistencia de legislación profesional; aprendizaje no reglado, por lo que se sustentan en una transmisión oral de madres a hijas, y e) no regulación de sus condiciones de trabajo

3. Para regular el número de personas dedicadas al marisqueo y garantizar una gestión controlada del recurso la Administración exige contar con un Permiso de Explotación Marisquera (PERMEX), cuya obtención depende de la situación en la que se encuentran las distintas especies de marisco. Asimismo, su renovación, que se realizan anualmente, está condicionada por el cumplimiento de una serie de requisitos: horas y días de dedicación, tareas, etc.

Feminismo/s 33, junio 2019, pp. 171-196 
ni de las enfermedades provocadas por su exposición a las inclemencias del tiempo, a las posturas forzadas y a los movimientos repetitivos.

En consecuencia, el reconocimiento social reflejaba esta descripción y las mariscadoras tenían mala fama, como personas problemáticas y conflictivas (Marugán, E colleron; Santasmarinas). Una calificación que también tiene relación con una sanción simbólica devenida de ubicarse (y dejarse ver) en un lugar que no les correspondía por género (Marugán, La organización 90). Es decir, de ocupar un espacio público (las playas) conocidos como «territorios de frontera, como son las riberas de las rías gallegas» (Broullón Acuña, La política sexual 83).

En definitiva, se vivía una situación de absoluto descontrol (Santasmarinas) donde las playas soportaban el marisqueo de unas 12.000 personas, de las cuales se calcula que el $80 \%$ no estaba dado de alta en la Seguridad Social (Marugán, E colleron). Esto repercutía en las condiciones de unas trabajadoras cuyo salario medio rondaba los 1.200 euros al año, lo que no hacía rentable el marisqueo (Marugán, E colleron). Además, como tampoco contaban con visión comercial, vendían fuera de lonja, haciéndose competencia entre ellas y supeditando sus ventas al deseo de los compradores con la consecuente repercusión en el precio de su producto.

Pero esta falta de concienciación y la consecuente desregulación del sector también afectaba al medio ambiente, pues provocaba una autodepredación de los recursos y una fuerte dependencia institucional para repoblar y vigilar las playas. Como no había cultura de inversión, se cogía el marisco sin el tamaño comercial mínimo, vaciando los arenales, ignorando los periodos de veda para preservar la reproducción de las especies.

Esta situación general influye también en su participación en las cofradías de pescadores ${ }^{4}$. Como extractoras de recursos, las mariscadoras siempre han podido participar como socias en estos espacios, a diferencia de los colectivos feminizados en tareas de apoyo. Sin embargo, y con excepciones, pocas veces se ejercía este derecho. Justificándose en los bajos ingresos que generaban,

4. Las cofradías de pescadores son entidades de derecho público que gestionan el sector pesquero, según los recursos existentes en cada zona. En la actualidad, en Galicia existen 63, con sus correspondientes federaciones provinciales en A Coruña, Lugo y Pontevedra y la común a todo el territorio (Federación Galega de Confrarías).

Feminismo/s 33, junio 2019, pp. 171-196 
en muchas cofradías se dificultaba la participación de las mujeres y no tenían presencia en la toma de decisiones (Mahou). Además, tampoco tenían agrupaciones de mariscadoras que defendieran sus intereses sectoriales.

Esta falta de influencia se extendía también a las instituciones que, en sus intentos de regular el marisqueo, las excluían como interlocutoras y solo hablaban con los patrones mayores, principal cargo de las cofradías y que normalmente eran hombres ligados a las embarcaciones. Hizo falta un cambio en la manera de enfrentar la desorganización y la marginalidad de estas mujeres, promovida por unos agentes técnicos imbuidos en la práctica participativa y en la perspectiva de género (Martínez-García, Democratizando el mar; Martínez-García y Aguado-Peláez) que transformara las formas de hacer institucionales e hiciera protagonistas a las propias mujeres, hasta ese momento nunca tenidas en cuenta 5 .

Así que, en conjunto, las mariscadoras a pie encajan en las tres formas de injusticias a las que se refiere el modelo tridimensional de Nancy Fraser y su propuesta de paridad participativa para revertirlas (Scales; Fortunas): obstáculos económicos y materiales en el ámbito distributivo; las trabas culturales y sociales en la esfera del reconocimiento, y exclusiones políticas y falta de influencia en la representación. Como veremos someramente, el proceso protagonizado por las mariscadoras a pie incide en estas tres cuestiones y se han convertido en un referente de organización en el mundo de la pesca tras un largo proceso de profesionalización que se inicia en los años 90.

De la mano de una política de regularización impulsada por la Xunta de Galicia, han transformado su oficio de un ejercicio meramente extractivo a otro en el que también se involucran labores de cultivo, gestión, comercialización, limpieza o vigilancia. Esta transformación ha influido en la racionalización y en la mejora de sus condiciones laborales; en el incremento de sus ingresos y en el control de la gestión de trabajo; en la organización del sector y en su capacitación, así como en el acceso a las estructuras de poder (Mahou; Marugán, E colleron).

5. En esta transformación, una figura central fue la jefa del Servicio de Extensión Pesqueira, Prudencia Santasmarinas, recientemente fallecida. Esta técnica jugó un papel fundamental en el proceso protagonizado por las mariscadoras a pie, primero, y que luego fue heredado por las rederas. 
Así, este artículo se incorpora a estos esfuerzos por visibilizar las experiencias de las mariscadoras a pie gallegas. Y, con ello, se adentra en las vivencias de estas mujeres que han ido ganando, progresivamente, cuotas de poder en sus comunidades.

\section{METODOLOGÍA}

Este trabajo forma parte de una investigación más amplia en la que se examina el vínculo entre procesos de gobernanza y empoderamiento de las mujeres del mar gallegas y vascas (Martínez-García, Empoderamiento femenino). En el mismo, se detectan unas tensiones entre las mariscadoras (y las trabajadoras del mar en general) que se refieren a: (1) su condición de mujeres en una sociedad patriarcal que precariza sus trabajos e invisibiliza sus aportaciones, y (2) su condición de mujeres marineras en un entramado que desvaloriza sus comunidades y formas de vida (Martínez-García, Mirando al mar). A partir de este marco, el presente artículo se centra en analizar las experiencias en cargos de poder de las mariscadoras a pie en Galicia dentro de las organizaciones de la pesca.

Para ello, se hace uso de dos herramientas fundamentales para comprender la complejidad y el dinamismo social: la interseccionalidad y la matriz de dominación de Patricia Hill Collins (Black Feminist). Por un lado, la primera permite detectar cómo interrelacionan los distintos ejes de opresión (Crenshaw). En el caso de las mariscadoras a pie: el género, la clase y su condición laboral, la edad y su posición de mujeres rurales-marineras ${ }^{6}$, su origen gallego y el vínculo familiar por su ligazón con el mar, así como el nivel formativo.

La combinación de estos ejes posibilita una mirada grupal, pues, sin una pretensión homogeneizadora, se entiende que conforman un colectivo desde

6. Las mujeres que se han entrevistado en este trabajo ligan sus experiencias rurales a entornos pesqueros. Una clasificación que responde a núcleos poblacionales más pequeños pero que, en este trabajo, se encuentra más vinculado a una idea de lugar definida por «la combinación y la coincidencia de un conjunto de relaciones socioespaciales» (McDowell 147). En el caso de las áreas costeras-marineras en Galicia nos encontramos con una fuerte identidad comunitaria, valores tradicionales pero más progresistas que en territorios de interior, escasez de servicios públicos acompañada de una sensación de abandono por parte de las instituciones, de las que se desconfía (Lorenzo y Martínez).

Feminismo/s 33, junio 2019, pp. 171-196 
las vivencias acumuladas por sus diferentes respuestas a desafíos compartidos (Collins, Black 28). Unos desafíos que, en el caso de las mariscadoras a pie, se mueven en la zozobra de conseguir mejoras (en torno a condiciones laborales, reconocimiento social e igualdad de oportunidades) en una situación general marcada por la incertidumbre en la pesca.

Esto nos lleva a la segunda herramienta que se corresponde con el marco de interpretación que se recoge en este monográfico, ya que el trabajo de Collins (Black Feminist) nos ayuda a ver cómo se organizan estas opresiones en un contexto determinado y cómo se desarrollan las relaciones de poder en: (1) el ámbito estructural, relativo a las políticas públicas que organizan y regulan las instituciones sociales; (2) el disciplinario, que administra y gestiona las relaciones de poder; (3) el hegemónico/cultural, que involucra las prácticas sociales que producen conciencia y valores (y contribuye a interiorizarlos), y (4) el interpersonal, referido al espacio personal, las vivencias con otras personas y su relación con la comunidad.

En definitiva, la matriz ayuda a explicar cómo la dominación, organizada a nivel macro y micro, evoluciona y es una realidad dinámica, en la que conviven formas de opresión y resistencia en el mismo colectivo dependiendo del momento y el lugar (Collins, Black Feminist 277-287). Todo un entramado que es fundamental tener en cuenta para hablar de procesos de profundización democrática en clave de justicia social (Martínez-Palacios y Martínez-García).

A partir de aquí, con el fin de observar estas dinámicas y analizar las experiencias de las mariscadoras en su acceso al poder, se hace uso de una metodología cualitativa. En este sentido, se utilizan dos recursos de análisis: a) la legislación y documentación relacionadas con las mujeres de la pesca que permite abordar el nivel macro -cabe recordar la ausencia de estudios y estadísticas sobre los colectivos feminizados que perpetúa la ocultación de las aportaciones de estas mujeres a las comunidades (Durán; Pérez Orozco, La economía) - y b) entrevistas en profundidad a mariscadoras a pie ${ }^{7}$ que permiten abordar el nivel experiencial.

7. Como se ha mencionado con anterioridad, este artículo se enmarca en un trabajo más amplio sobre los cambios vividos por las mujeres del mar a partir de su profesionalización. En este sentido, aunque solo se extraen verbatim de las entrevistas más 
Respecto a esto último, se realizan entrevistas en profundidad a seis mariscadoras a pie que ocupan (o han ocupado) un cargo en organizaciones sectoriales en la provincia de Pontevedra. Para su selección, se hace uso de un muestreo intencional que busca responder a la heterogeneidad de sus voces (ver tabla 1). En este sentido, el perfil es el de mujer gallega de entre 40 y 50 años -salvo una excepción de una mariscadora jubilada por su rico conocimiento sobre el proceso de profesionalización- y con diferente concienciación de género, lo que implica diversas formas de (no) identificar opresiones y de generar estrategias alternativas más o menos confrontativas. Respecto al nivel formativo, las de mayor edad tienen menos estudios y las más cercanas a los 40 años cuentan con titulación media. Por último, salvo una de ellas, todas tienen un vínculo familiar directo con la pesca por parte de padre y/o madre. La excepción que se menciona se inicia en el mar a través del esposo y su familia.

Tabla 1. Entrevistas a Mariscadoras

\begin{tabular}{|l|l|}
\hline M1 & $\begin{array}{l}\text { Patrona mayor de una cofradía en Pontevedra. Cargo en federación provincial } \\
\text { y gallega }\end{array}$ \\
\hline M2 & Ex-presidenta de una Agrupación de Mariscadoras en Pontevedra \\
\hline M3 & $\begin{array}{l}\text { Vicepatrona en una Cofradía en Pontevedra. Presidenta de Agrupación de } \\
\text { mariscadoras }\end{array}$ \\
\hline M4 & Ex-Patrona mayor de una cofradía en Pontevedra. Percebeira. \\
\hline M5 & Patrona mayor de una cofradía de Pontevedra. Percebeira. \\
\hline M6 & $\begin{array}{l}\text { Patrona mayor de una cofradía en Pontevedra. Cargo en Anmupesca y } \\
\text { Presidenta de Agrupación de mariscadoras }\end{array}$ \\
\hline
\end{tabular}

Fuente: Elaboración propia

directamente relacionadas con el objetivo de este artículo, se considera importante mencionar el trabajo de campo más amplio en el que se realizaron un total de 40 entrevistas a agentes técnicos y trabajadoras en Galicia y Euskadi (mariscadoras, rederas, empacadoras y neskatillas). A este trabajo también se suma un dossier de prensa de unas 650 noticias ligadas al proceso de las mariscadoras, 230 al de las rederas y 65 a colectivos vascos, y el análisis documental de informes, legislaciones y planes ligados a la actividad pesquera y a la igualdad en los distintos niveles territoriales.

Feminismo/s 33, junio 2019, pp. 171-196 


\section{DISCRIMINACIONES Y RESISTENCIAS EN EL MAR}

Las transformaciones que las mariscadoras a pie han experimentando desde que se inició el proceso de profesionalización en los años 90 las ha obligado a enfrentarse a múltiples dificultades. En este sentido, se reproduce un pequeño extracto del documental El cielo es nuestro techo. Historia de las mariscadoras de Cedeira, escrito y dirigido por Carlos Cazurro. Este documental, financiado por crowfunding, recoge las experiencias de las mariscadoras que participaron en la creación de la agrupación en este puerto de A Coruña. Dos de las protagonistas, Carmen y Teresa, comentan lo siguiente:

- [Carmen]: Porque aquí era una cofradía muy machista y a las mujeres nos querían ver desde muy lejos. Nos insultaban, nos llamaban berberecheiras, nos decían de todo.

- [Teresa]: No teníamos derecho ninguno: podíamos vender y nos quitaban el tanto por ciento y nada más. Pero hoy somos socias como un hombre, tenemos el mismo derecho que un marinero.

- [Carmen]: Nos llamaban de todo. Nos llamaban lo peor, lo peor... Y hoy quieren ser lo peor. Porque hoy cada mujer que va a la playa, con lo que hemos conseguido, puede vivir dignamente, no con lujos, pero sí dignamente, de lo que gana.

El caso de las mujeres de Cedeira es uno de los ejemplos más conflictivos que se vivieron en Galicia durante la profesionalización del marisqueo. Los problemas procedían de dos cambios fundamentales, como se desprende de las palabras de Carmen y Teresa. Por un lado, la entrada de las mujeres en un mundo de hombres como eran las cofradías de pescadores. Y, por otro, la extensión de otra forma de marisquear más controlada en la que solo pueden acceder a extraer los recursos de las playas quienes tienen permiso para hacerlo. Frente a estas dificultades, las mariscadoras contaban con el apoyo de la Xunta de Galicia y los agentes técnicos, que estaban decididos a actuar ante la degradación del medio marino y las pérdidas económicas de un sector tan rico, en un marco europeo desde el que se impulsaban políticas de igualdad (Mahou; Martínez-García, Empoderamiento femenino).

Así, la introducción de un enfoque participativo, horizontal y con perspectiva de género fue fundamental para promover la implicación de las mariscadoras en este proceso de profesionalización (Mahou; MartínezGarcía, Democratizando el mar; Empoderamiento femenino; Martínez-García 
y Aguado-Peláez; Marugán, E colleron; La organización). Un proceso que se fundamentó en tres pilares: la transformación de una actividad basada en la extracción y la explotación a una cultivadora y comercial; la formación, y la organización. Una intervención pública que ha acarreado importantes mejoras. Sin ánimo de exhaustividad, se mencionarán algunas.

Según la REMSP (Diagnóstico de la situación... en el marisqueo), los ingresos aumentaron de forma generalizada, si bien la renta generada depende del territorio y la situación de las especies: desde los $920 € /$ mes en Cambados hasta los 400 en Carril. Asimismo, se ven reconocidas como profesionales, consiguiendo ciertos derechos laborales como el coeficiente reductor o las ayudas cuando no pueden ejercer su actividad. Por otro lado, se crean agrupaciones de mariscadoras en todas las cofradías con presencia del marisqueo a pie para defender sus intereses. Las mujeres acceden a los puestos de poder: de las dos patronas mayores que había en 1995 se pasa a once tras las elecciones de 2018, con un 25\% de representación en los cargos directivos de las cofradías (cabildos).

Además, se han incorporado con un papel relevante a la Asociación Nacional de Mujeres de la Pesca (Anmupesca), cuya presidenta es una mariscadora a pie de Arcade. Además, otras organizaciones como los Grupos de Acción Local Pesqueira (GALP) cuentan con una presencia de mujeres, y de mariscadoras a pie en particular, muy relevante. Especialmente, en la promoción de proyectos de diversificación de los que el 48,8\% de las iniciativas son presentadas por mujeres. Muchas de ellas reconocidas como buenas prácticas de emprendimiento y sostenibilidad (REMSP, Buenas prácticas).

Estos movimientos han venido acompañados de un mayor reconocimiento social, comunitario e institucional, con un aumento de actos de homenaje y participación en jornadas marineras. Y, todo ello, ha devenido en un empoderamiento individual y colectivo, con un aumento de autoestima y confianza, de compañerismo, de acción colectiva y de influencia en el entorno (Martínez-García, Empoderamiento femenino).

No obstante, pese a las constantes mejoras experimentadas durante los últimos años, este sector sigue marcado por la incertidumbre. Por un lado, el furtivismo sigue presente, lo que influye en la reducción de la productividad de las playas. Una productividad que también se ve afectada por la contaminación y la crisis ecológica global que, entre otros efectos, calienta las aguas,

Feminismo/s 33, junio 2019, pp. 171-196 
repercutiendo en la calidad y cantidad de marisco (REMSP, Diagnóstico de la situación... en el marisqueo). Por otro lado, muchas de las políticas públicas que se impulsan desde la Unión Europea están más centradas en la diversificación turística que en el mantenimiento y potenciación de la pesca artesanal, lo que ahoga las formas de vida de estas trabajadoras (Martínez-García, Mirando al mar).

En consecuencia, ante las bajas expectativas de futuro, el relevo generacional es preocupante. Según el IGE, en el año 2018, de las mujeres con Permex, casi el 85\% tienen más de 40 años (el 39,4\% se encuentra en la franja entre 51 y 60) y solo el 15\% tiene menos -si nos fijamos en las de menos de 30 años, el porcentaje se reduce al 3\%-. Todas estas circunstancias afectan también a las experiencias de las protagonistas de este artículo, no solo por su condición de mariscadoras, sino por su posición de liderazgo en las organizaciones del sector.

\subsection{Trabas y zancadillas en las organizaciones pesqueras}

El acceso de las mariscadoras a los espacios de poder no ha sido fácil. Y la ocupación de cargos de responsabilidad en las cofradías ha estado, en la mayoría de los casos, impregnada de dolores y tensiones, aunque también de resistencias y apoyo mutuo. A continuación, se analizarán las discriminaciones experimentadas por las mariscadoras entrevistadas, así como las estrategias generadas en este proceso.

En los inicios, la creación de las agrupaciones de mariscadoras fue fundamental para reforzar su presencia y voz colectiva en unas cofradías que, en la mayoría de los casos, ignoraban las demandas y necesidades de las mujeres $^{8}$. No obstante, las presiones que tuvieron que vivir fueron muchas, procedentes de los hombres y de quienes tenían la costumbre de coger el marisco sin control:

Cuando se creó la agrupación hubo mucho debate y enfrentamientos con gente que estaba acostumbrada a andar no mar. Parecía que la culpa la teníamos nosotras cuando eran normas que había que aplicar (M5).

8. M1 tiene una posición diferenciada de sus compañeras a este respecto y entiende que las agrupaciones en su cofradía han cogido demasiado poder y necesitan ser controladas para pensar en un interés general.

Feminismo/s 33, junio 2019, pp. 171-196 
La obligatoriedad de aplicar las normas convivía con un sentimiento de culpa y de responsabilidad, ya que muchas veces tenían que limitar el trabajo de sus compañeras. Una carga personal que se acompañaba del esfuerzo por parte de las impulsoras para gestionar todo el proceso, más duro todavía al tener, en muchos casos, una baja formación. Como explica una de ellas:

No había estudiado leyes, ni yo ni ninguna de mis compañeras de la directiva, era un mundo totalmente desconocido, tenías que leer muchos papeles, había palabras que no entendía, tenías que mirar qué significa esto... [...] $\mathrm{Y}$ luego las reuniones con las mujeres para explicarles y aquello era una batalla campal (M2).

Aun así, una de las peores consecuencias de este proceso fue el enfrentamiento con aquellos grupos privilegiados que, tradicionalmente, habían dirigido las cofradías. Unos enfrentamientos que no se diluyeron en los 90, sino que en muchos casos se reproducen todavía ahora. De manera que, aunque ha habido avances y cada vez es mayor la legitimidad de estas mujeres, todavía perviven muchos envistes patriarcales. Cuatro de las seis entrevistadas manifiestan haber experimentado resistencias, en muchos casos violentas, que tienen que enfrentar con los medios a su alcance. En ocasiones, encuentran en la normativa oportunidades para sobrevivir, como se refleja en el siguiente testimonio:

Conseguimos redactar un Régimen interno muy duro, con sanciones fuertes para los furtivos y que garantizaran el ejercicio de la actividad en igualdad. Fue un trabajo muy duro ya que tenía que estar todo bien documentado y tuvimos que aprender y movernos. Creamos un listado de infracciones y, claro, atacaba prácticamente a los hombres porque eran los infractores. Pero aunque fue una avance enorme a nivel organizativo, creó todavía un odio ${ }^{9}$ mayor hacia las mujeres que le decían a los hombres que tenían que cambiar su forma de trabajar» (M4).

9. El odio del que habla esta mariscadora se tradujo en una moción de censura que la revocó del cargo en favor de una gestora. No obstante, los números le dieron la razón y en las últimas elecciones contó con el apoyo mayoritario de las compañeras y compañeros que, salvo unos pocos, la reeligieron como patrona. Además, es importante tener en cuenta que, en el ejercicio del trabajo de percebeira se depende mucho de las otras personas por el riesgo que se corre, así que «cuando nos dicen que si nos ven ahogarnos no se echan a ayudarnos» (M4) se puede entender directamente como una amenaza.

Feminismo/s 33, junio 2019, pp. 171-196 
También experimentaron múltiples zancadillas para frenar su actividad. Una de ellas, que se dedica al percebe (uno de los recursos más lucrativos del sector), relata algunas de estas trabas: pagar más impuestos, poder extraer menos kilos de recurso y ser las últimas en vender, siempre después de los hombres e independientemente de la calidad del producto (M4). Por otro lado, en algunas cofradías los patrones mayores se negaban a prestar su apoyo en épocas de dificultad. Otra mariscadora cuenta la respuesta de su compañero en un momento de crisis por una riada, en el que necesitaba su firma para solicitar una subvención:

Decía que el dinero tenía que ser para la Cofradía y no para las mujeres. Hablaba por detrás, nos boicoteaba [...] Es como si nos tuvieran miedo, porque a lo mejor tenemos el poder. En mi cofradía somos mayoría mujeres así que si nos vamos a vender a otro lado se arruina. En otros sitios igual. Así que piensan que es mejor tenernos controladas (M6).

Aun así, mayormente también señalan que, a nivel de Cofradía y en el trato con la Administración, se han debilitado mucho las desigualdades. Y reconocen que muchos de sus compañeros han ido aprendiendo y valorando tanto su presencia como la introducción de nuevas formas de gestionar las cofradías:

Es una presión enorme, pero poco a poco muchos han aprendido y entendido cosas [...] Las mujeres (algunas) eran mi apoyo, mi fuerza... Porque empezamos a ver cómo vendíamos al mismo tiempo, en los mismos espacios, todos los percebeiros fuera de la zona de subastas, pidiendo turnos para pesar la mercancía y sin empujones. [...] A pesar de los enfrentamientos, que todavía algunos continúan porque solo piensan en sus intereses, hemos conseguido ir educándolos en un respeto hacia las mujeres del sector, de que teníamos los mismos derechos (M4).

Antes se hacía una cena de navidad para mujeres y otra para hombres. Ahora hacemos una todos juntos (M6).

No obstante, al ascender en la escala de poder, la posición en las federaciones no es tan confortable y no se sienten tan protegidas por el compañerismo local. En la Federación Galega, el presidente y los dos vicepresidentes son hombres. Y de los 12 miembros del comité ejecutivo, 4 por federación provincial, solo hay dos mujeres en A Coruña, la vicepatrona mayor de Muros y la de Pontedeume. Esta falta de presencia femenina se corresponde con una 
mayor incomodidad, expresando una sensación de ser tratadas con condescendencia o poco respetadas cuando hablan. Así que:

Te vas haciendo respetar y te hablan como a un hombre sino te tratan como tontitas (M1).

\subsection{Camuflarse en un mundo de hombres}

Como se observa de la cita anterior, el respeto se gana cuando se aprende a participar en estos espacios según los mandatos hegemónicos (Martínez Palacios, Innovaciones democráticas) que otorgan reconocimiento y legitimidad a ciertas presencias y voces consideradas normativas (Juliano, Young). Una normatividad que representa al sujeto privilegiado (en este contexto, un hombre blanco con carisma y experiencia) y que, disfrazada de neutralidad, encierra las lógicas de dominación masculinas. Así, muchas veces, cuando las mujeres quieren ser escuchadas asumen estos comportamientos. Estrategia que siguen muchas de las entrevistadas:

En este mundo de hombres yo aprendí muy rápido que, o me volvía como ellos, me movía como ellos, hablaba como ellos y me hacía escuchar como ellos o no aguantaba ni una semana (M4).

En una primera reunión salí asustada porque había hombres que tenían dos huevos, ¿eh? Pero tú puedes tener la costumbre de hablar mal, cuando me enfado, pero a veces tengo que ponerme brava. Que soy mujer, pero mira... Y soltar un hostia para que se escuche (M1).

Otra cuestión a tener en cuenta es la conciliación con los trabajos domésticos y el cuidado de crianzas y personas mayores, ya que las patronas mayores son una figura de autoridad social muy importante a nivel local y se les exige presencia en muchos eventos, además de la gestión de las cofradías. Sus testimonios evidencian lo que, desde el feminismo, se viene denunciando: la incompatibilidad de sostener la vida según los modelos productivos vigentes (Carrasco; Herrero, Pascual y González Reyes; Izquierdo; Pérez-Orozco, Subversión feminista). Sirvan de ejemplo:

No tengo vida (y eso que no tengo hijos) (M4).

Es una trampa porque siempre recae en nosotras. La vida cómoda es la de ellos, yo si volviera a nacer no sé si querría ser hombre, que viven mejor. 
[...] Además, acabamos perdiendo pasos en las decisiones, ya que ellos lo acaban de arreglar todo tomando una cerveza (M6).

En este orden de cosas, una de las protagonistas, madre de un hijo pequeño, cuenta que muchas veces tenía que llevarse al niño a las reuniones porque no podía afrontar el pago continuo de ludotecas:

Me joroba que muchos sábados tenemos que hacer reuniones, sobre todo en la gallega y la provincial, y movidas por las tarde que no me dejan estar con él. Puedes rechazar algunas cosas, pero no puedes decir todo el rato que no vas (M1).

En este sentido, la presencia de mujeres, aunque no es definitiva, es estratégica para moldear otro tipo de organizaciones y contribuye a visibilizar el trabajo oculto de cuidados y los espejismos del actual modelo de conciliación (Carrasco; Pérez Orozco, Subversión).

\subsection{Oportunidades para otra acción política}

La creciente presencia de mujeres en las cofradías ha posibilitado problematizar las formas de organización y de gestión, así como los tiempos y necesidades de las personas que participan en estas entidades. Asimismo, también ha derivado en una mayor asunción de la diversidad de intereses, del diálogo y la negociación frente a modos más verticales e intolerantes de decisión que se daban en algunos de estos espacios. Como explica una de ellas:

No tengo problemas en hablar y tiro de ellos y me dan ideas, porque soy consciente de que quieren jubilarse donde están y van a defender lo suyo. Hay que dialogar, oír, hay que saber estar [...]. Hablar con todos. Tengo que defender el sector (M6).

En este sentido, sus habilidades para la negociación son un importante recurso en el cargo de patrona, estableciendo un equilibrio entre las diferentes demandas y movilizando la solidaridad entre los distintos colectivos. Cuentan:

En el trabajo tenemos que estar todos unidos y ser compañeros y luego te vas de copas con quien quieras (M1).

Intento que lleguen a acuerdos. Si estuviera como presidenta de la agrupación [del marisqueo a pie] iba a ser más dura, pero no puedo porque yo como patrona necesito que trabajen todos los sectores (M4).

Feminismo/s 33, junio 2019, pp. 171-196 
Así, frente a un deseo de aglutinar poder, estas patronas mayores buscan compartirlo y generar respuestas colectivas que concilien todos los intereses que existen en una cofradía:

¿Quiénes somos nosotras para gestionar la vida de 200 familias? Hay que tener mucha cabeza y mucha responsabilidad. Porque de las decisiones que tomamos dependen muchas personas (M6).

Esta predisposición a los acuerdos y a la negociación también se encuentra en su pretensión de generar redes más allá del contexto local y del marisqueo. Un ejemplo es el caso de Anmupesca, un hito organizativo en el ámbito de la pesca cuya firma se realiza el 19 de mayo de 2016 por parte de 12 colectivos de mujeres ${ }^{10}$. En esta organización conviven, no sin conflictos, trabajadoras de varios subsectores relacionados con la pesca y con diversas posiciones sociales. Frente a posiciones de algunas armadoras que pedían que las mujeres que ejercieran cargos directivos tuvieran formación universitaria, la respuesta de las mariscadoras no se hizo esperar, reivindicando la importancia de espacios seguros y horizontales para todas. Describen su respuesta:

Yo soy una mujer de la pesca, voy todos los días al mar, sé lo que es tener lumbagos y dolores, sé sobre accidentes laborales. Si quisiéramos montar una asociación de gente con carrera, la montábamos. Pero para montar una asociación de burócratas ya tenemos bastante. Y ante estas palabras, reaccionaron [...] Vamos a intentar abrirnos a que participen todas las mujeres posibles y a partir de ahí ya iremos congeniando (M6).

Estas palabras son de especial interés para reivindicar el derecho de los grupos oprimidos a la autodefinición (Collins Black 69-123). Al igual que, en su momento, las agrupaciones de mariscadoras fueron una fuente de apoyo y

10. De Galicia: las Asociaciones de Mariscadoras Virxe do Carme de Arcade, Río Anllóns y Mulleres do Mar de Poio; las agrupaciones de rederas O Fieital de Malpica, O cerco de Cangas do Morrazo, Illa da Estrela de Corme y do Baixo Miño Atalaia de A Guarda; la Asociación de Frigoríficos de Pesca Extractiva AFRIPEX, y la Organización de Productores de Pesca de Palangre ORPAL. Desde la Comunidad Valenciana: la Asociación de Armadores de Santa Pola y, por Andalucía: la Asociación de Mujeres de la Pesca Galatea y Venux Nux. Información disponible sobre sus orígenes, trayectoria y asociadas actuales en: <http://www.anmupesca.org/>. Es importante señalar que las Cofradías, como organizaciones, no pueden participar en la Asociación, lo que ha generado el rechazo de alguna de las entrevistadas (M1, M5).

Feminismo/s 33, junio 2019, pp. 171-196 
de reforzamiento del papel de estas trabajadoras en la cofradía, reclaman la importancia de significarse como mujeres del mar:

Damos la información de primera mano, hablamos nosotras por nosotras ¿Meten la pata los políticos y no vamos a meterla nosotras que somos del sector primario? A lo mejor nos pierden las maneras pero nos van a entender igual y nosotras defendemos lo nuestro (M6).

En esta defensa de lo suyo, entienden que la solidaridad y la creación de redes entre ellas es fundamental. Sentencia una de las protagonistas:

Yo pienso que hay que luchar juntas, todas tenemos una historia con el mar. Y cuanto más nos reunimos, cuanto más nos entendemos, más ilusión se crea $[\ldots]$ Nosotras vamos a aprender hablando, saliendo, mirando, abriendo la mente (M6).

Y cuidando el entorno y a quienes lo habitan, reconociendo el trabajo invisible de muchas de sus predecesoras. Unos valores que no solo aplican a su actividad tradicional como extractoras, sino también a proyectos de diversificación económica en los que están inmersas algunas de ellas. Concluye una mariscadora que el éxito de sus experiencias se encuentra en:

El amor por nuestra actividad principal y fuente de ingresos. Y también en la sensibilidad por dar a conocer y hacer visible el trabajo de las mujeres en el mar, en dar a conocer nuestra cultura y, muy importante, en el respeto y cuidado del medio ambiente como fuente de riqueza y salud (M3).

\section{CONCLUSIONES}

Dice Antonio Luances en su columna de opinión en el Faro de Vigo publicada el 28 de julio de 2014, titulada «Las mujeres pisan fuerte»:

Aunque ha sido mucho de lo que se ha hablado del papel de la mujer en el mundo de la pesca y el marisqueo gallegos, más queda todavía por escribir de los importantes cambios que la mujer propicia cuando accede al cargo de patrona mayor de las cofradías. [...] Es seguro que la nueva sensibilidad puede hacer que el mundo de las cofradías cambie definitivamente.

Así, la incorporación de las mujeres a la dirección y gestión de las cofradías ha alterado el panorama pesquero. Como se ha descrito, estos cambios se pueden resumir en los siguientes: una formas de actuar más horizontales, equitativas y transparentes; unas pretensiones más relacionales e interactivas, y una actitud 
más abierta y conciliadora. A pesar de todas las dificultades experimentadas, son conscientes de que todas y todos son importantes para sostener la actividad pesquera y sus comunidades. De esta manera, las mariscadoras a pie $y$, en general, las mujeres que acceden a espacios de poder en el sector pesquero, se conforman como un sujeto transformador a la hora de generar nuevas oportunidades en el mar. Como mujeres en un mundo de hombres, son capaces de incorporar otra mirada en el ejercicio de las responsabilidades políticas y sociales en las organizaciones pesqueras. A su vez, como mujeres marineras guardan una importante solidaridad con sus compañeros, con los que conforman un sector económico cada vez más marginal. Esta condición les proporciona una comprensión mayor de las necesidades de sus comunidades y aporta una perspectiva más amplia que la apuesta única por la diversificación turística impulsada por las instituciones europeas. Además, como trabajadoras que dependen del entorno, tienen una importante sensibilidad ecológica que las ayuda a equilibrar la sostenibilidad del medio ambiente con la supervivencia económica.

Por lo tanto, las experiencias de las mariscadoras a pie se constituyen como una fuente de aprendizaje para afrontar los retos del contexto actual, porque estas mujeres se han ido incorporando a las diferentes organizaciones y han impulsado rupturas con formas de hacer que, no por rutinarias, son menos injustas. Han promovido otras maneras de organizarse y gestionar las entidades, más eficaces y transparentes. Y han sido capaces de establecer redes con otros territorios y con otros sectores, buscando espacios de encuentro entre intereses diversos. En otras palabras, son capaces de «moverse en la tensión creativa entre lo deseable, lo posible, lo probable y lo práctico» (Collins, A difference 24) para avanzar hacia la justicia social en el mar. Y es que no podemos olvidar que las mariscadoras son expertas en encontrar tesoros bajo pequeños granos de arena. 


\section{REFERENCIAS BIBLIOGRÁFICAS}

Amorós, Celia. Hacia una crítica de la razón patriarcal. Madrid: Anthropos Editorial del Hombres, 1985.

-. Tiempo de feminismo. Sobre feminismo, proyecto ilustrado y postmodernidad. Madrid: Ediciones Cátedra, 2000.

Broullón, Esmeralda. «Culturas marítimas y relaciones de poder. La trayectoria el marisqueo a pie en las Rías Bajas gallegas». Cuadernos de Estudios Gallegos 123 (2010): 375-399.

—. «La política sexual y la segregación ocupacional en las sociedades pesqueras». Revista de Estudios Feministas, Florianópolis 19 (2011): 73-90.

Carrasco, Cristina. «El cuidado como eje vertebrador de una nueva economía». Cuadernos de Relaciones Laborales 31.1 (2013): 39-56.

Cazurro, Carlos. «El cielo es nuestro techo. Historia de las mariscadoras de Cedeira». 2017. 30 de mayo de 2019.

Collins, Patricia Hill. Black feminist thought. Knowledge, consciousness, and the politics of empowerment. Segunda edición: New York: Routledge, 2000.

—. «The difference that power makes». Investigaciones feministas 8.1 (2017): $19-40$.

Crenshaw, Kimberlé. «Mapping the margins. Intersectionality, identity, politics, and violence against women of colour». Stanford Law Review 43.6 (1991): 1241-1299.

Del Valle, Teresa. Mujer vasca. Imagen y realidad. Barcelona: Anthropos Editorial del Hombre, 1985.

Durán, María-Ángeles. «El desafío económico de las mujeres». Sociología y género. Eds. Capitolina Díaz Martínez y Sandra Dema Moreno. Madrid: Editorial Tecnos, 2013. 183-199.

Federación Galega de Confrarías de Pescadores. As mulleres do mar en Galicia. Situación actual das profesións tradicionais desempeñadas por mulleres no ámbeto pesqueiro-conserveiro. A Coruña: Federación Galega de Confrarías de Pescadores, 2008.

Fraser, Nancy. Scales of Justice. Reimagining Political Space in a Globalizing World. Cambridge: Polity Press, 2008.

- Dilemas de la Justicia en el siglo XXI. Género y globalización. Palma de Mallorca: Universitat de les Illes Balears, 2011.

—. Fortunas del Feminismo. Madrid: Traficantes de Sueños, 2015. 
Fundación Mujeres. ¿Contra viento y marea? Mujeres en el sector de la pesca. Guía para el profesorado. Incorporación de la perspectiva de género a la formación continua del sector de la pesca de altura. Madrid: Fundación Mujeres, 2006.

Gago, Constantino y Ardora Formación. A muller no mundo da pesca en Galicia. A Coruña: Xunta de Galicia, 2004.

García Negro, María do Carme y Yolanda Zotes. «El trabajo de las mujeres en el sector pesquero gallego: análisis de los problemas relacionados con su tratamiento estadístico». Revista Galega de Economía 15 (2006): 1-25.

Juliano, Dolores. Tomar la palabra. Mujeres, discursos y silencios. Barcelona: Ediciones Bellaterra, 2017.

Herrero, Yayo, Marta Pascual y María González Reyes. La vida en el centro. Voces y relatos ecofeministas. Madrid: Libros en Acción, 2018.

Izquierdo, María Jesús. «Del sexismo y la mercantilización del cuidado a su socialización: Hacia una política democrática del cuidado». Cuidar cuesta. Costes $y$ beneficios del cuidado. Actas del Congreso Internacional Sare 2003. Ed. Ana Rincón. Vitoria-Gasteiz: Emakunde, 2004. 120-154.

Lorenzo, Ana y Miguel Martínez. "Condiciones y experiencias de participación en la Galicia rural». Praxis participativa desde el medio rural. Eds. Javier Encina, M. ángeles Ávila, Manuela Fernández y Montse Rosa. Madrid: Iepala Editorial-Cimas, 2003. 301-320.

Luances, Antonio. «Las mujeres pisan fuerte». Faro de Vigo, 28 de julio de 2014. Mahou, Xosé M. Implementación y gobernanza. La política del marisqueo en Galicia. Santiago de Compostela: Xunta de Galicia, Escola Galega de Administración Pública, 2008.

Marçal, Katrine. ¿Quién le hacía la cena a Adam Smith? Una historia de las mujeres y la economía. Barcelona: Debate, 2016.

Martínez-García, Patricia. «Democratizando el mar con perspectiva de género. El proceso de profesionalización de las mariscadoras a pie en Galicia». Política y Sociedad 54.2 (2017): 365-386.

-. Empoderamiento femenino en contextos de gobernanza. Diss. Universidad del País Vasco / Euskal Herriko Unibertsitatea, 2017.

-. «Mirando al mar desde los feminismos. Comunidad y supervivencia en torno a las trabajadoras de la pesca». Prisma social 19 (2017): 183-214.

Martínez García, Patricia y Delicia Aguado Peláez. «Democratizar la participación con una mirada feminista. La importancia de los agentes técnicos en la profesionalización de las mujeres de la pesca en Galicia». Participar desde los 
feminismos. Ausencias, expulsiones y resistencias. Ed. Jone Martínez Palacios. Barcelona: Icaria, 2017. 209-234.

Martínez Palacios, Jone. Innovaciones democráticas feministas. Editorial Dykinson: Madrid, 2018.

Martínez Palacios, Jone y Patricia Martínez-García. «Introducción. Justicia social, interseccionalidad y profundización democrática». Investigaciones feministas 8.1 (2017): 9-17.

Marugán, Begoña. E colleron ese tren... Profesionalización das mariscadoras galegas. A Coruña: Xunta de Galicia, 2004.

-. «Estrategias laborales ante los desafíos ecológicos globales. Valorizar socialmente las contribuciones de las mujeres del sector pequero español para asegurar la pervivencia del oficio». La Ventana 17 (2003): 107-139.

—. «La organización de las mariscadoras como agentes de transformación social». Em Debate 7 (2012): 82-106.

McDowell, Linda. Género, identidad y lugar. Madrid: Ediciones Cátedra, 2000. Pateman, Carole. El contrato sexual. Barcelona: Editorial Anthropos, 1995.

Pérez Fuentes, Pilar. "Ganadores de pan» y «Amas de casa». Otra mirada sobre la industrialización vasca. Bilbao: Servicio Editorial de la UPV-EHU, 2004.

Pérez Orozco, Amaia. «La economía: de icebergs, trabajos e (in)visibilidades». Transformación del trabajo desde una perspectiva feminista. Producción, reproducción, deseo, consumo. Ed. Laboratorio Feminista. Madrid: Tierra de Nadie Ediciones, 2006. 233-253.

-. Subversión feminista de la economía. Aportes para un debate sobre el conflicto capital-vida. Madrid: Traficantes de Sueños, 2014.

Red Española de Mujeres en el Sector Pesquero (REMSP). Buenas prácticas emprendedoras promovidas por mujeres en el sector pesquero. Madrid: Ministerio de Agricultura, Pesca y Alimentación, 2016.

-. Diagnóstico sobre la situación profesional de las mujeres en el oficio del marisqueo en España. Madrid: Ministerio de Agricultura, Pesca y Alimentación, 2016.

- Diagnóstico sobre la situación de la mujer en el sector pesquero y acuícola. Madrid: Ministerio de Agricultura, Pesca y Alimentación, 2017.

Salas, Elena y Nieves Salobral. «Antielectras, esquizofrenia y marginalidad». Transformación del trabajo desde una perspectiva feminista. Producción, reproducción, deseo, consumo. Ed. Laboratorio Feminista. Madrid: Tierra de Nadie Ediciones, 2006. 125-136. 
Santasmarinas, Prudencia. « ¿Tiene futuro el marisqueo?». Revista Galega de Economía 15.1 (2006): 1-5.

Vega Solís, Cristina, Raquel Martínez-Buján y Myriam Paredes Chauca. Cuidado, comunidad y común. Extracciones, apropiaciones y sostenimiento de la vida. Madrid: Traficantes de Sueños, 2018.

Young, Iris Marion. Inclusion and democracy. Oxford: Oxford University Press, 2002. 